Revista Signos

2010, 43(72)

$99-123$

\title{
Documentos profesionales con destinatarios no expertos. El empleo de los mecanismos referenciales en la sentencia del $11 \mathrm{M}^{1}$
}

\author{
Anna López Samaniego \\ Universidad de Barcelona \\ España
}

Resumen: El objetivo de este artículo es analizar una de las sentencias judiciales que ha tenido mayor repercusión en la historia de España: la que juzgó los atentados terroristas del 11 de marzo de 2004 en Madrid. El interés lingüístico de esta sentencia reside en que está redactada por un emisor especialmente consciente de que su texto iba a tener una gran difusión en medios no solo jurídicos, sino también de comunicación de masas. La hipótesis de partida es que estas circunstancias de producción excepcionales permiten considerar la sentencia del $11 \mathrm{M}$ como exponente de un intento real de redactar una sentencia judicial de comprensión accesible para el destinatario no experto. El estudio de esta sentencia se centra en el análisis cuantitativo y cualitativo de uno de los principales aspectos lingüístico-discursivos que evidencian el esfuerzo de claridad discursiva realizado por el juez ponente: el manejo de las expresiones demostrativas anafóricas ('esto', 'este fenómeno'...). El análisis de este mecanismo referencial en la sentencia del $11 \mathrm{M}$ se contrasta con el del mismo mecanismo en otras seis sentencias emitidas por el mismo órgano jurisdiccional, la Audiencia Nacional. Las conclusiones arrojan luz acerca del papel que puede desempeñar el Análisis del Discurso Profesional en una posible modernización del discurso judicial en español.

Palabras Clave: Sentencia judicial, inteligibilidad, optimización del discurso profesional, pronombres demostrativos, sintagmas nominales anafóricos.

Recibido:

1-III-2009

Aceptado:

30-IX-2009
Correspondencia: Anna López Samaniego (alopezsam@ub.edu). Departamento de Filología Hispánica, Sección de Lengua, Facultad de Filología, Universidad de Barcelona. Edifici Històric, Gran Via de les Corts Catalanes 585, 08007, Barcelona, España. 


\title{
Professional documents for a non-expert audience. The use of mechanisms of reference in the March 11 court sentence
}

\begin{abstract}
The aim of this paper is to analyze one of the court sentences that has had a great impact on Spanish History: the sentence on the case of the terrorist attacks of March 11, 2004, in Madrid. The linguistic interest of this sentence is that its author was especially aware that the text would be widely disseminated not only in the legal field but also in the mass media. Our hypothesis is that due to this exceptional context, the M11 court sentence is an example of a real attempt to write a legal text comprehensible to a non-expert audience. Our study of this court sentence focuses on the quantitative and qualitative analysis of a linguistic aspect that clearly shows the effort made by the judge pronouncing the sentence to ensure clarity: the use of anaphoric demonstrative expressions ('this', 'this phenomenon'...). The analysis of this mechanism of reference in the M11 court sentence is contrasted with the same mechanism in six other sentences passed by the same court, the Audiencia Nacional (The High Court). The findings shed light on how Professional Discourse Analysis could contribute to the modernization of Spanish judicial discourse.
\end{abstract}

Key Words: Legal sentences, readability, professional discourse-optimization, demonstrative pronouns, anaphoric nouns.

\section{INTRODUCCIÓN²}

La sentencia judicial es un tipo de resolución emitida por un juez que tiene como funciones comunicativas: (i) resolver las pretensiones y solicitudes de las partes implicadas en un proceso (Alcaraz \& Hughes, 2002); (ii) comunicar tal resolución a las partes afectadas; y (iii) justificar la decisión judicial en función de principios propios del Derecho. Se trata, por lo tanto, de un texto informativo, ya que resume los datos más relevantes de un proceso judicial y, sobre todo, argumentativo y directivo, ya que comunica y justifica decisiones que afectan directamente a las partes implicadas. Dada la importancia de la dimensión perlocutiva de la sentencia judicial, la redacción de estos documentos profesionales se encuentra sometida a una serie de requisitos.

Para empezar, al igual que ocurre en muchos otros textos profesionales como los textos instruccionales o los textos destinados a la atención al cliente, la sentencia judicial está sometida a un imperativo de claridad, fundamentalmente porque las sentencias son textos profesionales con un destinatario lego en Derecho, el ciudadano. En el caso de la sentencia, además, el requisito de accesibilidad del texto tiene también una motivación de índole propiamente jurídica: el deber de garantizar mediante una argumentación judicial clara que la resolución del juez se adecua a la legislación vigente.

Como se desprende de las líneas anteriores, la sentencia es un género cuya redacción se en- 
cuentra condicionada por el 'dilema de la doble audiencia' (two audience dilemma): se trata de un texto con un doble destinatario, especialista (juristas) y no especialista (las partes afectadas). El dilema consiste en que si el juez intenta acercar su lenguaje a las partes -que constituyen, en teoría, el alocutor o destinatario directo de la sentencia-, pierde credibilidad profesional entre sus colegas de profesión, que participan también en la comunicación en tanto que destinatarios indirectos o participantes ratificados ${ }^{3}$. Por el contrario, si opta por mantener su discurso especializado, falla la comunicación con las partes afectadas (Gibbons, 2003).

Esta segunda opción, la de contar con un destinatario experto y mantener la imagen autorizada como profesional, suele ser la mayoritaria, a juzgar por las críticas habituales de los especialistas, tanto juristas como lingüistas, que suelen calificar el discurso judicial de oscuro, críptico y ambiguo (de Miguel, 2000; Bayo, 1996, 2002). Las críticas a la opacidad judicial son frecuentes, pese a que el artículo 218 de la Ley de Enjuiciamiento Civil (LEC 1/2000) estipula que las sentencias deben ser "claras, precisas y congruentes".

No obstante, otro aspecto especialmente idiosincrásico de la sentencia frente a otros tipos de discurso profesional es que, en ocasiones, los textos judiciales no solo resultan ambiguos o incomprensibles para el destinatario no experto, sino también para el experto, tal como han indicado los propios juristas (Bayo, 1996; Ordóñez, 2002). Entre las inconsistencias discursivas que se han identificado como causa de la opacidad discursiva de la sentencia judicial en español destacan: (i) recurrentes errores de normativa tanto morfosintáctica como ortográfica; (ii) la construcción de períodos oracionales excesivamente dilatados (Campos, 2007); como consecuencia de lo anterior, (iii) la abundancia de oraciones subordinadas, encadenadas mediante numerosos incisos, cláusulas absolutas y gerundios, que permiten condensar información (Alcaraz \& Hugues, 2002); y, finalmente, (iv) graves problemas de puntuación que vienen a agravar los problemas de comprensión producidos por los rasgos ya mencionados. Todo ello conlleva a menudo ambigüedades sintácticas y rupturas de la estructura de la oración, esto es, anacolutos (Montolío \& López Samaniego, 2008).

La existencia de textos jurídicos de interpretación costosa, cuando no imposible, no es exclusiva del lenguaje jurídico español. Por este motivo, desde la década de los años 70 se han ido sucediendo los movimientos internacionales que defienden la necesidad de acercar el discurso jurídico al ciudadano. En España, y también en otros países de habla hispana que han iniciado más recientemente este proceso -como México, Argentina o Chile-, las reformas se han limitado al ámbito jurídico de la Administración, ya que es en este en el que resulta más evidente el diálogo directo con el ciudadano 4 .

Sin embargo, en el ámbito de la Administración de Justicia española tales movimientos se 
encuentran todavía en estado incipiente. Es cierto que el Gobierno expresó en la Carta de Derechos de los ciudadanos ante la Justicia (propuesta no de ley, enmarcada en el Pacto de Estado para la Reforma de la Justicia de 2001 y aprobada por el Congreso de los Diputados en 2002) su intención de modernizar y hacer accesible al ciudadano el lenguaje de la Justicia 5 . Desde ese momento, el Gobierno ha reiterado en algunas ocasiones su compromiso de poner en marcha una reforma del lenguaje judicial, pero hasta el momento no se han llevado a cabo más medidas concretas al respecto (Montolío \& López Samaniego, 2008).

\section{La sentencia 'mediática' del 11M}

En el contexto descrito de la intención de reformar el lenguaje judicial español, el 31 de octubre de 2007 se dicta la sentencia del $11 \mathrm{M}$, que resuelve el juicio por los atentados terroristas del 11 de marzo de 2004 en Madrid. Se trata de una sentencia excepcional por una serie de motivos: en primer lugar, su extensión (721 folios); en segundo lugar, el número de participantes (28 procesados, 309 testimonios, 71 peritos, 51 abogados, 4 fiscales, 3 jueces); en tercer lugar, el volumen de información recopilada (310 horas de vista oral, 100.000 folios de sumario de instrucción judicial); en cuarto lugar, su relevancia internacional, ya que se trata de la primera sentencia en condenar el terrorismo yihadista en Europa; y, por último, su repercusión socio-política en España, donde esta sentencia representó el esclarecimiento de los hechos que dieron lugar a la llamada 'teoría de la conspiración', que había constituido motivo de enfrentamiento en la política española de los últimos años ${ }^{6}$.

Todas estas características explican que existiera una gran expectación social tanto en España como en el ámbito internacional ante la publicación de esta sentencia. En este sentido, si bien en otras sentencias el 'dilema de la doble audiencia' descrito en el apartado anterior suele resolverse en favor de la audiencia especializada, esto es, de los juristas; la sentencia del 11M representa un ejemplo de resolución judicial redactada con una marcada conciencia de la diversidad de los destinatarios. Entre dichos destinatarios destacaban, en este caso, las partes afectadas, la clase política, y el conjunto de la sociedad en general, obviamente a través de los medios de comunicación, que difundieron diversos fragmentos de la sentencia original.

En definitiva, se trata de una sentencia escrita con una especial conciencia de la heterogeneidad de los destinatarios, tanto expertos como no expertos. La previsible amplia difusión del texto, así como la ingente cantidad de información que este manejaba, permiten suponer que el redactor de la sentencia, el magistrado Gómez Bermúdez, invirtió un esfuerzo especial en potenciar la claridad expositiva de su texto. Este esfuerzo se comprende aún más si se tiene en cuenta que entre las finalidades de la sentencia figuraba también establecer la verdad oficial 
de los hechos ocurridos el 11 de marzo de 2004, por lo que convenía no dejar lugar a ambigüedades a las que pudieran seguir asiéndose los defensores de la teoría conspirativa.

Por todas estas circunstancias, este artículo parte de la hipótesis de que el análisis lingüísticodiscursivo de la sentencia del $11 \mathrm{M}$ puede revelar cuáles de los rasgos consustanciales al discurso jurídico que suelen observarse en las sentencias redactadas en español y que, por lo general, dificultan su comprensión ${ }^{7}$, son prescindibles en favor de la claridad expositiva, sin que por ello quede desvirtuado el carácter especializado propio de un texto jurídico.

\subsection{Presentación del corpus y la metodología}

A fin de corroborar la hipótesis de partida, nos centramos en el análisis de uno de los mecanismos lingüístico-discursivos que facilitan la comprensión de la sentencia del 11M: los demostrativos anafóricos. Para demostrar que el juez del $11 \mathrm{M}$ emplea estos mecanismos referenciales de forma especialmente eficaz, contrastamos el empleo de los demostrativos anafóricos en la sentencia del $11 \mathrm{M}$ con su uso en otras seis sentencias de los años 2004 y 2006, redactadas por distintos jueces de la misma Sala de lo Penal de la Audiencia Nacional.

Dada la extensión de los corpus manejados, el análisis se ha acotado al apartado de los Fundamentos de Derecho, en el que (i) se recogen los hechos probados, que en algunas otras sentencias suelen establecerse en un apartado independiente; (ii) se exponen los argumentos que garantizan que la decisión alcanzada por el juez se basa en la aplicación del Derecho; y (iii) se extrae la ratio decidendi, esto es, el principio jurídico fundamental que determina la decisión y que puede llegar a constituir jurisprudencia para casos posteriores (Bhatia, 1993). Este es el fragmento nuclear (y más extenso) de toda sentencia. Así, los fundamentos jurídicos de la sentencia del $11 \mathrm{M}$ constan de 85.349 palabras, mientras que los de las seis sentencias de la Audiencia Nacional suman 84.473 palabras.

Ambos corpus son comparables, puesto que comprenden textos similares en más de una lengua o variedad (Maia, 2003). En nuestro caso, además de compartir un número de palabras y una situación comunicativa similar, las sentencias que conforman el segundo corpus se seleccionaron en función del tipo de proceso, con múltiples procesados, a fin de que fuera equiparable al de la sentencia del $11 \mathrm{M}$ en cuanto al uso de los mecanismos referenciales.

En cuanto a las distintas variedades lingüísticas que se comparan, se trata aquí de comparar el estilo de un texto emitido en unas condiciones circunstanciales que requieren un especial cuidado del lenguaje, la sentencia del $11 \mathrm{M}$, con otros textos que suelen producirse habitualmente en el mismo órgano jurisdiccional (la Audiencia Nacional, Sala $2^{\mathrm{a}}$ ). 


\section{Mecanismos de organización sintáctica y discursiva en la sentencia del 11M}

En un primer análisis de aproximación al texto de la sentencia del 11M se corroboró la intuición inicial acerca de la mayor lecturabilidad o accesibilidad al significado de esta sentencia y se identificaron los principales rasgos que contribuyen a ello. Se trata, especialmente, de rasgos relativos a la organización del texto y a la sintaxis discursiva.

En primer lugar, en la organización global del texto destaca, por una parte, la marcación de la estructura de la sentencia mediante un total de 46 epígrafes organizados en una serie de títulos y subtítulos que avanzan el tema de cada apartado mediante un sistema constante y coherente de numeración. El empleo de estos títulos facilita tanto la localización rápida de la información como la comprensión de la estructura temática o macroestructura global del texto (van Dijk, 1978). Por otra parte, predomina también claramente en la sentencia del $11 \mathrm{M}$ el párrafo breve, con muchas enumeraciones que rompen con frecuencia la linealidad gráfica del texto, evitando así la oración-párrafo, característica del estilo de las sentencias (Alcaraz \& Hugues, 2002).

En cuanto a este último punto, cabe indicar que en la sentencia que nos ocupa las enumeraciones, muy frecuentes en el discurso judicial y que a menudo presentan una marcación escasa o incoherente (López Samaniego, 2006), aparecen por lo general marcadas. Dicha marcación se concreta en el empleo de expresiones predictivas (Tadros, 1985), ordenadores del discurso que introducen los elementos de la serie, o incluso mediante ambos recursos, como puede observarse en los siguientes dos ejemplos:

(1) El Tribunal Constitucional (...) para comprobar si ha existido una real posibilidad de defensa contradictoria exige que se respeten tres reglas:

a) Nadie puede ser acusado sin haber sido, con anterioridad, declarado judicialmente imputado.

b) Como consecuencia de lo anterior, nadie puede ser acusado sin haber sido oído con anterioridad a la conclusión de la investigación.

c) No se debe someter al imputado al régimen de las declaraciones testificales, (...), ya que la imputación no ha de retrasarse más allá de lo estrictamente necesario (SAN 65/2007, ff. 442-443) ${ }^{8}$.

(2) Respecto de Abdelmajid BOUCHAR y los atentados de Madrid del 11 de marzo, Moussaten declaró dos cosas: una, que su tío Youssef BELHADJ le dijo que Abdelmajid BOUCHAR había encontrado el camino [para huir de España] y que Dios le había ayudado, la otra, que estaba de acuerdo con los atentados, que le parecía poco lo que había pasado (SAN 65/2007, ff. 641-642).

Por lo que respecta a la sintaxis discursiva de la sentencia del $11 \mathrm{M}$, nos parece destacable la 
abundante inclusión de breves paréntesis aclaratorios, que desempeñan funciones reformulativas dirigidas a evitar una posible 'fuente de perturbación' (Gülich \& Kotschi, 1995): (i) repeticiones que retoman elementos que han aparecido previamente en el texto, pero que podrían haber desaparecido de la memoria a corto plazo de los lectores (ejemplo 3); (ii) denominaciones que aclaran el significado de un fragmento previo (4); o (iii) paráfrasis que aseguran el correcto proceso inferencial del lector acerca de las relaciones entre los elementos referidos en el texto (5):

(3) Y si las partes lo que sostienen es que se han producido relevantes irregularidades en la cadena de custodia que priva a la bolsa con el explosivo de la condición de fuente de prueba obtenida con todas las garantías, provocando una tacha insubsanable de nulidad de esta pieza de convicción a efectos de prueba, con los efectos que de ello derivan, están aceptando la preexistencia del objeto (bolsa con explosivo) y su relación con el delito (SAN 65/2007, f. 512).

(4) De ellos, 3 fueron retirados el mismo día de la compra y otros 6 fueron encargados para recogerlos una vez manipulados electrónicamente para poder ser usados con cualquier operador de telefonía -en argó liberados- al día siguiente (SAN 65/2007, f. 529).

(5) A su vez, el número 652282947 -recordemos, el del soporte plástico encontrado en Chinchón-es uno de los 30 anteriormente citados en el punto 4, también vendidos al locutorio de la calle Tribulete (SAN 65/2007, f. 522).

En relación con la frecuencia de operaciones textuales de aclaración, es también elevada la presencia de reformulaciones expansivas que introducen explicaciones que a veces incluso rayan en la redundancia, como en el ejemplo (6). En él se expresa un mismo contenido con otras palabras hasta un total de cuatro veces, sin añadir demasiada información nueva -excepto la subrayada-, tal como indicamos numerando las repeticiones:

(6) ... [el dolo radica en el conocimiento del peligro concreto que la conducta desarrollada supone para el bien jurídico, en este caso, vida (entre otras muchas SS.T.S. de 1 de diciembre de 2004, 14 de febrero de 2005 y la citada de 15 de febrero de 2006) $]^{12}$.

Dicho de otro modo, [el dolo criminal implica el conocimiento de la significación antijurídica del hecho $\mathrm{y}$, a la vez, la voluntad para realizarlo $]^{2^{2}}$. [Exige capacidad cognoscitiva y volitiva] $]^{3}$.

[El dolo directo existe cuando, de manera consciente y querida (capacidad cognoscitiva), la voluntad del sujeto se dirige directamente al resultado propuesto (capacidad volitiva), incluidas las consecuencias necesarias del acto que se asume $]^{4 \mathrm{a}}$ (SAN 65/2007, ff. 487-488). 
Cabe indicar que la reiteración de contenidos es habitual en los textos jurídicos y, en concreto, en la sentencia judicial, ya que, en ocasiones, lo que puede parecer redundante o repetitivo para el no experto, es realmente necesario para evitar la ambigüedad en beneficio de la precisión. No obstante, esta posibilidad de ambigüedad o de imprecisión suele darse sobre todo en relación con los conceptos técnicos jurídicos, como el de ‘dolo' descrito en el ejemplo anterior, pero es mucho más difícil que aparezca cuando los juristas manejan palabras o expresiones propias del léxico común. De ahí que en la sentencia del 11M resulten especialmente sorprendentes las aclaraciones relativas a expresiones comunes empleadas en la descripción de los hechos (premisas fácticas):

(7) A preguntas de la defensa de Zougam y Ghalyoun añadió este funcionario que vio diversos objetos dentro de la furgoneta, pero que no eran significativos, por lo que no les prestó mayor atención y que desde fuera había ángulos muertos; o sea, partes del interior no visibles desde el exterior (SAN 65/2007, f. 506).

Fragmentos como los anteriores muestran el esfuerzo realizado por el juez del $11 \mathrm{M}$ para controlar todas las inferencias realizadas por los lectores de su sentencia, es decir, para confiar las mínimas inferencias posibles sobre el contenido del texto a la libre interpretación del destinatario.

Ahora bien, para demostrar que, efectivamente, la sentencia del 11M presenta una redacción presidida por una conciencia del destinatario no experto mayor que la habitual, es necesario llevar a cabo un análisis de la sintaxis del documento en mayor profundidad, que demuestre que existen en esta sentencia rasgos que facilitan la interpretación del texto y explique su funcionamiento. Por ello, nos centramos a continuación en el análisis de uno de estos rasgos: el uso de los demostrativos anafóricos.

\section{Los pronombres y sintagmas demostrativos anafóricos}

La elección del objeto de estudio, los mecanismos textuales anafóricos, responde a diversas razones. Como ya se ha mencionado en la introducción, uno de los requisitos estilísticos de la sentencia judicial consiste en evitar cualquier ambigüedad o posibilidad de que exista más de una interpretación sobre una misma expresión. Sin embargo, tal como ha afirmado Kurzon (1997), la anáfora es, junto con la coordinación ${ }^{9}$ y la modificación, una de las principales causas de ambigüedad en los textos jurídicos. De hecho, algunos de los principales especialistas en el discurso jurídico anglosajón han puesto de relieve que, aunque la presencia de pronombres es inevitable en los textos escritos, los profesionales del Derecho -y, en especial, los legislado- 
res- suelen preferir la repetición léxica al uso de pronombres, a fin de evitar la ambigüedad de referentes (Tiersma, 1999; Gibbons, 2003). ${ }^{10}$

No obstante, a pesar de todas estas precauciones, lo cierto es que la identificación ambigua de la referencia de un pronombre en algunos de los textos jurídicos anglosajones se ha aprovechado para dar origen a litigios (Solan, 1993). El riesgo de ambigüedad que suele comportar el empleo inadecuado de estas expresiones es, pues, una de las causas para la elección de este objeto de estudio.

El segundo motivo para la elección de las expresiones anafóricas radica en que se trata de un mecanismo fundamental en la construcción del sentido del mensaje, ya sea como mecanismo de cohesión, por cuanto recupera necesariamente un elemento que ya ha aparecido en el discurso previo; ya sea como elemento básico para la construcción de la coherencia del texto; o incluso como procedimiento de progresión de la información, pues facilita la continuidad temática, reactivando el referente (o recuperándolo de la memoria a corto plazo del lector) y, a menudo, ampliando información sobre este.

\subsection{Anáforas, pronombres demostrativos y nombres anafóricos}

Como su etimología indica, las expresiones anafóricas son 'repeticiones' de palabras, expresiones, oraciones o incluso párrafos que ya han aparecido en un discurso previo. La relación entre la expresión anafórica y la expresión previa a la que se refiere (el antecedente) es, por tanto, de correferencia o identidad de referentes. Ello explica que, para interpretar las expresiones anafóricas, sea necesario o bien recuperar el referente en la memoria a corto plazo, o bien volver la vista atrás en el texto para localizarlo. La anáfora es, por tanto, una relación de referencia estrictamente textual.

Dado que el repertorio de expresiones anafóricas en español es extenso, ya que incluye los pronombres personales y demostrativos, sintagmas nominales definidos, el sujeto elíptico, etc., en este artículo nos centramos en el análisis de las expresiones demostrativas con valor anafórico.

Frente a otras expresiones que pueden desempeñar funciones anafóricas, los demostrativos presentan una peculiaridad: a la identificación del antecedente añaden la localización deíctica de este: "sitúan algún elemento del contexto de la enunciación con respecto al lugar en el que se encuentra el hablante” (Eguren, 1999: 936).

Los pronombres demostrativos pueden remitir, por tanto, a una entidad presente o implicada en el contexto de la enunciación, con un referente exofórico, externo al texto (como en 'Tú, 
cállate ahora mismo'). No obstante, el empleo de los pronombres demostrativos que interesa a este análisis es el anafórico, aquel en el que el referente se encuentra dentro del propio discurso. En dicho empleo anafórico, los demostrativos localizan el referente y pueden indicar también la posición del antecedente respecto de la expresión anafórica (cercanía/lejanía). Este empleo endofórico del demostrativo suele ser el más frecuente en los textos escritos -más descontextualizados que los orales- y especialmente en los textos especializados.

La selección de las distintas expresiones anafóricas suele explicarse en la bibliografía más reciente por las instrucciones que cada una de ellas proporciona al interlocutor para identificar el antecedente. Ariel $(1988,1990)$ se ha referido a dichas instrucciones con el concepto de 'accesibilidad'. Se trata de un concepto jerárquico que designa el grado de disponibilidad del referente en el contexto o en la memoria (enciclopédica o de trabajo) del interlocutor. Según se desprende de las escalas de accesibilidad más citadas, cuanto más semánticamente vacía se encuentra una expresión referencial, menos contribuye por sí misma a la identificación del referente (Ariel, 1988, 1990; Gundel, Hedberg \& Zacharski, 1993).

De acuerdo con este marco teórico, resulta esperable que, cuando el emisor considera que el antecedente de una expresión anafórica es poco accesible o difícil de recuperar, seleccione una expresión anafórica más informativa, por ejemplo, un sintagma definido ('el primer acusado'). Por el contrario, si el emisor considera que el antecedente es accesible, tenderá a seleccionar una expresión anafórica menos informativa ('él, este, $\varnothing$ ').

A su vez, la elección de una expresión con grado alto o bajo de accesibilidad depende de la prominencia (salience) del antecedente (von Heusinger, 2007), que viene marcada por factores como (i) la distancia entre la expresión y su referente, (ii) el tipo de estructura sintáctica en la que expresión y referente se introducen o (iii) el conocimiento contextual y enciclopédico que se le presupone al interlocutor. Los problemas de interpretación que suelen plantear las expresiones anafóricas ocurren, pues, como parece de esperar, cuando el emisor realiza un "cálculo erróneo" de la accesibilidad de la expresión empleada (Figueras, 1996: 67).

Según la teoría de la accesibilidad de Ariel (1990), los pronombres demostrativos, como pronombres que son, presentan un grado de accesibilidad baja: imponen una serie de restricciones de uso mayor que la que requieren otras expresiones anafóricas más informativas. Por este motivo, es frecuente que en los textos escritos -y, aún más, en los especializados- cuando se emplea un demostrativo para realizar una referencia retrospectiva a un elemento previo del texto, el emisor sienta la necesidad de añadir junto a él un nombre que o bien repite el antecedente literalmente, o bien constituye un sinónimo, cuasi-sinónimo o hiperónimo de este. Esta necesidad de emplear un elemento anafórico con mayor informatividad o mayor contenido 
léxico se intensifica cuando el antecedente se encuentra más lejos que la frase anterior o cuando hay más de un antecedente posible en el discurso previo (Ariel, 1990).

El mayor grado de informatividad que caracteriza las expresiones anafóricas compuestas por un demostrativo y uno de estos nombres anafóricos (p.e. 'este fenómeno') ${ }^{11}$ facilita la interpretación del referente, a diferencia del uso de un mero pronombre demostrativo (este). En los sintagmas del tipo <demostrativo + nombre anafórico>, el demostrativo aporta la función deíctica de dirigir la atención del destinatario hacia una determinada parte del cotexto, mientras que el nombre aporta información que facilita la interpretación semántica unívoca del antecedente (Lyons, 1980). Así, los sintagmas nominales anafóricos contribuyen a cohesionar la cadena expositiva, a la vez que la hacen avanzar, reactivando y, a menudo, reformulando elementos previos.

Aunque el determinante que acompaña a estos nombres anafóricos puede ser también un artículo definido, estos sintagmas con valor anafórico presentan una acusada tendencia a aparecer con demostrativos o elementos similares (Conte, 1996). Asimismo, dichos sintagmas pueden aparecer complementados por modificadores o calificadores (Francis, 1994) que, en ocasiones, pueden poseer mayor relevancia informativa para la identificación del referente que el propio nombre anafórico (Flowerdew, 2003).

\subsection{Los demostrativos anafóricos en los textos especializados}

Teniendo en cuenta las características gramaticales del español, parece previsible que la frecuencia de aparición de las expresiones pronominales anafóricas en esta lengua sea más elevada que en inglés. De hecho, así lo indica un reciente estudio contrastivo realizado sobre textos científicos: si bien el inglés presenta una mayor tendencia a condensar información mediante la nominalización, el español tiende a condensar información tanto en nombres como en pronombres y adverbios (Álvarez de Mon y Rego, 2006).

En consonancia con estas afirmaciones, y aunque los estudios estadísticos sobre el empleo de mecanismos anafóricos en español son muy escasos, sí se ha reseñado la presencia habitual en los textos jurídicos en español de demostrativos y, en general, expresiones anafóricas como 'dicho', ‘mencionado', 'citado', 'expresado', 'este', 'ese' y 'aquel', etc. También es habitual en los textos jurídicos españoles el uso del pronombre 'ello' (Samaniego, 2005), a pesar de que presenta dificultades añadidas para la identificación del referente, puesto que por ser neutro carece de marcas de género y número.

Por lo que respecta al referente de las expresiones pronominales, este suele ser más extenso o denso en español que en inglés (Álvarez de Mon y Rego, 2006), especialmente por lo que res- 
pecta a los pronombres neutros ('ello', 'lo', ‘eso', etc.). Tales pronombres pueden aludir tanto a entidades de primer orden o físicas (personas, animales, objetos), como de segundo orden (acontecimientos, situaciones y estados de cosas) o también de tercero (proposiciones) (Eguren, 1999, empleando términos de Lyons, 1980). Esta amplia capacidad de referencia de los pronombres neutros, junto con la ausencia de marcas de género que faciliten la identificación del referente, dificulta especialmente la interpretación del antecedente de estas expresiones, por lo que su presencia en los textos especializados parece poco aconsejable.

Como conclusión de todo lo anterior, partimos de la hipótesis de que en la sentencia judicial serán más frecuentes las expresiones anafóricas informativas o de accesibilidad alta, por ejemplo, los sintagmas anafóricos ('este razonamiento'), que otras expresiones como los pronombres, dadas las características discursivas prototípicas de la sentencia judicial vistas en el primer apartado, como la frecuente intercalación de largos incisos o el predominio de períodos sintácticos extensos, que dificultan el acceso a los antecedentes. Veamos si tal hipótesis se cumple en las sentencias analizadas.

\section{Demostrativos anafóricos en la sentencia del 11M}

\subsection{Análisis cuantitativo}

La metodología de análisis aplicada ha consistido en la búsqueda automática de los demostrativos españoles ('este', 'ese' y 'aquel') en los dos corpus descritos en el apartado 1.1. La comparación cuantitativa de la frecuencia de uso de los demostrativos anafóricos en ambos corpus nos permitirá afirmar con un cierto fundamento empírico si realmente la situación comunicativa extraordinaria en la que se redactó la sentencia del 11M conllevó un uso especial de tales mecanismos por parte del juez que la redactó. Por su parte, la comparación cualitativa, mediante el análisis de algunos ejemplos, nos permitirá observar si el manejo de estas expresiones anafóricas en la sentencia del 11M está también presidido por un intento de facilitar la interpretación del texto y reducir las ambigüedades interpretativas; y, en caso de que así sea, observar en qué consiste exactamente tal manejo.

Puesto que los elementos analizados son de naturaleza textual y el propósito del análisis es comparar el estilo de los jueces, la búsqueda automática aplicada se ha combinado necesariamente con el filtro de la revisión manual de cada ocurrencia, a fin de seleccionar solo (i) los usos anafóricos de los demostrativos estudiados y (ii) las anáforas emitidas por el juez redactor de la sentencia. Por este motivo, se han excluido del estudio: 
a) Los escasos usos deícticos exofóricos, que no constituyen mecanismos de cohesión (Halliday \& Hasan, 1976), como suele ocurrir con los demostrativos de tercera persona ('aquel') o con expresiones propias del lenguaje judicial, como el empleo de 'este Tribunal' o 'este juzgador' para aludir a los emisores de la sentencia;

b) Anáforas intertextuales, es decir, incluidas en citas literales de lo dicho por otros emisores o en el contenido de documentos previos (generalmente de la fase de instrucción), ya que estas anáforas no constituyen elementos de cohesión manejados por el juez del 11M;

c) Pronombres demostrativos que se encuentran fosilizados en marcadores del discurso, como es el caso del demostrativo 'esto' en el reformulador 'esto es'. En cambio, sí se han incluido los casos en los que el pronombre eso aparece insertado en la estructura 'por eso', ya que esta todavía se encuentra en un estadio evolutivo intermedio hacia la conversión en marcador (Montolío, 2001). De hecho, la mayor parte de las ocurrencias del pronombre demostrativo eso son de este tipo.

Por otro lado, dado que el uso anafórico del pronombre demostrativo ‘aquello' suele limitarse a su uso combinado con esto para referirse al más lejano de dos posibles antecedentes (Figueras, 2000) y teniendo en cuenta que tal uso es poco habitual y no se da en las sentencias analizadas, hemos considerado más significativa la búsqueda del pronombre personal de tercera persona 'ello'. Es cierto que, a diferencia de los demostrativos, el pronombre 'ello' (i) es exclusivamente endofórico, (ii) no puede tener valor contrastivo, y (iii) puede tener un comportamiento distinto en determinados contextos (Fernández Soriano, 1999). No obstante, 'ello' -etimológicamente relacionado con 'aquello'- tiene un funcionamiento equivalente a los demostrativos neutros en su uso anafórico en la lengua escrita (Fernández Ramírez, 1987; Mederos, 1988; Álvarez Martínez, 1989), por lo que resulta de interés para los objetivos de este estudio.

Asimismo, añadimos a la búsqueda de pronombres demostrativos una expresión que en los textos jurídicos suele presentar el mismo valor que un pronombre demostrativo anafórico (de Miguel, 2000). Nos referimos al uso anafórico antinormativo (Real Academia Española 2005, s.v. “mismo-ma. 3.”) de las expresiones comparativas 'el mismo', 'la misma', 'los mismos', 'las mismas'.

Ofrecemos a continuación la Tabla 1, que recoge los resultados de la presencia de los pronombres demostrativos en los Fundamentos de Derecho de los dos corpus de sentencias analizados: 
Tabla 1. Presencia de los pronombres demostrativos en los corpus manejados.

\begin{tabular}{|l|c|c|}
\hline \multicolumn{1}{|c|}{ EXPRESIÓN ANAFÓRICA } & SENTENCIA DEL 11M & 6 SENTENCIAS A.N. \\
\hline este/a/os/as & 111 & 95 \\
\hline ese/a/os/as & 2 & 1 \\
\hline aquel/la/los/las & 6 & 27 \\
\hline esto & 23 & 17 \\
\hline eso & 6 & 10 \\
\hline ello & 37 & 45 \\
\hline $\begin{array}{l}\text { el mismo / la misma } \\
\text { los mismos / las mismas }\end{array}$ & 12 & 71 \\
\hline TOTAL & 197 & 266 \\
\hline
\end{tabular}

Una observación general a los datos de esta Tabla revela que la cantidad de pronombres demostrativos empleados parece algo superior en las sentencias de la Audiencia Nacional. Una lectura en mayor detalle permite observar, por una parte, que en la sentencia del $11 \mathrm{M}$ predominan los mecanismos de referencia pronominal de corto alcance y más concretos, como el demostrativo 'este', en todas sus variaciones de género y número, incluida la forma neutra; y, por otra, que el uso del falso anafórico ‘el mismo' es mucho menos frecuente en la sentencia del 11M que en el resto de las sentencias analizadas.

Asimismo, la frecuencia del neutro 'ello' es notablemente elevada en ambas sentencias, muy probablemente por su capacidad para referirse a conjuntos de conceptos o frases y por su frecuente combinación con conectores de finalidad ('para ello') o de causa retrospectiva ('por ello'). No obstante, los datos son todavía limitados y escasos como para conducir a conclusiones significativas.

Veamos ahora qué ocurre con los sintagmas con <demostrativo + nombre anafórico>. Cabe señalar que, además de los sintagmas compuestos con los demostrativos propiamente dichos, consideramos conveniente ampliar el estudio a sintagmas en los que el sustantivo anafórico aparece acompañado por determinantes como 'dicho' o 'tal', a los que las gramáticas suelen atribuir un valor muy similar al de los demostrativos anafóricos (Fernández Ramírez, 1987; Mederos, 1988; Eguren, 1999), ya que a menudo funcionan como meras variantes estilísticas de los anteriores. Los resultados de esta segunda búsqueda se sintetizan en la Tabla 2: 
Tabla 2. Presencia de sintagmas del tipo <demostrativo + nombre anafórico >

\begin{tabular}{|l|c|c|}
\hline \multicolumn{1}{|c|}{ EXPRESIÓN ANAFÓRICA } & SENTENCIA 11M & 6 SENTENCIAS A.N. \\
\hline este/a/os/as + nombre & 326 & 148 \\
\hline ese/a/os/as + nombre & 160 & 52 \\
\hline aquel/la/los/las + nombre & 6 & 21 \\
\hline tal/es + nombre & 28 & 164 \\
\hline dicho/a/os/as + nombre & 73 & 117 \\
\hline TOTAL & 593 & 502 \\
\hline
\end{tabular}

Esta segunda Tabla, además de corroborar nuestra hipótesis inicial de la mayor frecuencia de aparición de los sintagmas anafóricos respecto de las expresiones pronominales, arroja más luz sobre el uso de mecanismos anafóricos en las sentencias analizadas y proporciona muchos más aspectos dignos de observar en el análisis cualitativo, especialmente si lo combinamos con el anterior y obtenemos una visión de conjunto del uso de mecanismos deícticos con valor anafórico en ambos corpus:

Tabla 3. Presencia de demostrativos anafóricos en las sentencias analizadas.

\begin{tabular}{|l|c|c|}
\hline EXPRESIÓN ANAFÓRICA & SENTENCIA 11 M & 6 SENTENCIAS A.N. \\
\hline Pronombres demostrativos & 119 & 123 \\
\hline Pronombres demostrativos neutros & 66 & 72 \\
\hline $\begin{array}{l}\text { El mismo / la misma/ } \\
\text { los mismos / las mismas }\end{array}$ & 12 & 71 \\
\hline Demostrativo + nombre & 492 & 221 \\
\hline Tal/es + nombre & 28 & 164 \\
\hline Dicho/as/os/as + nombre & 73 & 117 \\
\hline TOTAL & 790 & 768 \\
\hline
\end{tabular}




\subsection{Análisis cualitativo}

Tal como demuestran las frecuentes críticas al estilo judicial, el escritor jurista no siempre es consciente, cuando emplea elementos anafóricos, de la necesidad de reactivar antecedentes alejados, difíciles de recuperar en la memoria de trabajo del lector. Es decir, no siempre realiza un cálculo correcto de la accesibilidad del antecedente que requieren las expresiones anafóricas seleccionadas. De hecho, los problemas de pérdida de referentes de las expresiones anafóricas son frecuentes en la sentencia judicial, especialmente cuando las expresiones seleccionadas son pronominales, como en el caso de 'el mismo' o 'este' (Montolío \& López Samaniego, 2006, 2008).

Así, en el ejemplo (8), procedente del corpus de sentencias de la Audiencia Nacional analizado, resulta complejo identificar el antecedente de las dos expresiones anafóricas destacadas, probablemente debido a la discordancia entre las expresiones anafóricas y el antecedente lógicamente más plausible ('la reunión'):

(8) El Sr. Francisco era sabedor que a las 20 horas del citado día 17 tenía una reunión en la Cámara de Comercio de Villagarcía y a la que, dada su condición de Vicepresidente, debía asistir, y más aun cuando éste era de importancia pues se iban a tratar temas de interés, como así lo manifiesta el Sr. Luis Antonio Secretario de la misma (SAN 4/2006).

El punto de partida de este artículo era la hipótesis de que el juez redactor de la sentencia del $11 \mathrm{M}$ había realizado un especial esfuerzo de claridad expositiva, motivado por las circunstancias de producción peculiares expuestas en el primer apartado de este estudio. Entre otros rasgos discursivos, sugeríamos que tal esfuerzo podía reflejarse en un empleo más eficaz del habitual de los mecanismos anafóricos, es decir, de una selección más consciente de las expresiones más adecuadas a cada contexto, con el fin de facilitar al máximo la identificación de los antecedentes.

Pues bien, el análisis de los datos de la Tabla 3 muestra, en primer lugar, que la cantidad de demostrativos anafóricos empleados en la sentencia del $11 \mathrm{M}$ es prácticamente la misma que la que se observa en un corpus de una extensión global muy similar, formado por 6 sentencias de tema equivalente, redactadas por seis jueces distintos de la misma jurisdicción. Sin embargo, resulta de interés comparar la diferente distribución de dichos mecanismos.

Para abordar el análisis cualitativo de las diferencias en el empleo de expresiones deícticas anafóricas en ambos textos, se precisa una visión completa y ordenada de los elementos empleados en cada corpus. Asimismo, a fin de que la comparación resulte más exacta, se han'normalizado’ 
los datos obtenidos para calcular la frecuencia de los distintos elementos anafóricos por cada 10.000 palabras (Biber, Conrad \& Reppen, 1998).

Tabla 4. Frecuencia de los demostrativos anafóricos en la sentencia del 11M.

\begin{tabular}{|l|c|}
\hline EXPRESIÓN ANAFÓRICA & TOTAL (frecuencia / 10.000 palabras) \\
\hline Demostrativo + nombre & $492(57.64)$ \\
\hline Pronombres demostrativos & $119(13.94)$ \\
\hline Dicho/as/os/as + nombre & $73(0.85)$ \\
\hline Pn. demostrativos neutros & $66(0.77)$ \\
\hline Tal/es + nombre & $28(0.32)$ \\
\hline $\begin{array}{l}\text { El mismo / la misma / } \\
\text { los mismos / las mismas }\end{array}$ & $12(0.14)$ \\
\hline TOTAL & $790(93.52)$ \\
\hline
\end{tabular}

Tabla 5. Frecuencia de los demostrativos anafóricos en 6 sentencias de la A.N.

\begin{tabular}{|l|c|}
\hline EXPRESIÓN ANAFÓRICA & TOTAL (frecuencia /10.000 palabras) \\
\hline Demostrativo + nombre & $221(26.16)$ \\
\hline Tal/es + nombre & $164(19.41)$ \\
\hline Pronombres demostrativos & $123(14.56)$ \\
\hline Dicho/as/os/as + nombre & $117(13.85)$ \\
\hline Pn. demostrativos neutros & $72(0.85)$ \\
\hline $\begin{array}{l}\text { El mismo / la misma / } \\
\text { los mismos / las mismas }\end{array}$ & $71(0.84)$ \\
\hline TOTAL & $768(90.91)$ \\
\hline
\end{tabular}

Ya se ha dicho en el tercer apartado de este artículo que las expresiones del tipo <demostrativo + nombre anafórico> facilitan al lector la recuperación de antecedentes poco accesibles, como los que se encuentran lejos de la expresión anafórica o los que 'compiten' con otro posible antecedente en el contexto inmediato. Del análisis comparativo entre las tablas anteriores se desprende que en ambos corpus, los sintagmas anafóricos (<demostrativo / tal / dicho + 
nombre>) son más frecuentes que las expresiones pronominales. Este resultado parece confirmar que la sintaxis discursiva propia de la sentencia judicial, caracterizada por la frecuente intercalación de incisos y por una excesiva extensión de las oraciones, potencia el uso de los mecanismos anafóricos de baja accesibilidad (como ‘este argumento').

No obstante, la frecuencia de aparición de los distintos mecanismos en ambos corpus es notablemente distinta. En la sentencia del 11M, los sintagmas anafóricos copan el 75\% de las expresiones anafóricas empleadas, frente al $65 \%$ que representan estos sintagmas en las sentencias de la Audiencia Nacional. Pero además, si se observa con mayor detalle, el 10\% de diferencia entre ambas sentencias no es tan significativo como las diferencias entre el tipo de sintagmas anafóricos empleados: en la sentencia del $11 \mathrm{M}$ solo los sintagmas < demostrativo + nombre anafórico> ocupan el $62 \%$ de los sintagmas anafóricos empleados, mientras que en las sentencias de la A.N., todos los sintagmas anafóricos empleados presentan una frecuencia de aparición mucho más equitativa: $28,7 \%$ para <demostrativo + nombre anafórico $>, 21,2 \%$ para <tal/es + nombre anafórico > y 15,1\% para <dicho/a/os/as + nombre anafórico $>$.

Con respecto al mayor número de ocurrencias de los sintagmas <demostrativo + nombre anafórico> en la sentencia del $11 \mathrm{M}$, cabe decir que, como ya se ha mencionado en este mismo apartado, la presencia del sustantivo anafórico suele facilitar la interpretación del antecedente. No obstante, el nombre anafórico no es el único elemento que aumenta la informatividad de las expresiones anafóricas en la sentencia del $11 \mathrm{M}$, sino que, si se atiende a un análisis cualitativo de los ejemplos, se observa que es frecuente además el uso de modificadores del nombre anafórico que evitan la posible ambigüedad en la interpretación del antecedente, tal como ocurre en los ejemplos (9) y (10):

(9) Éste [sic.] IMEI correspondía a un paquete de telefonía cuyo número de teléfono era el 652 28.29.47.

El soporte plástico correspondiente a esa tarjeta fue hallado en el registro de la finca de Chinchón. Y aquel terminal fue usado por Rachid Oulad Akcha -uno de los suicidas de Leganés- con la tarjeta con número 653026006 el día 9 de marzo de 2004.

Esta última tarjeta, la 653026006 , es una de las doscientas vendidas en packs Motorola C450 por ACOM a INTERDIST MÓVIL, S.L. el día 5 de febrero de 2004 (SAN 65/2007, ff. 519-520).

(10) El policía número 83.322, que vio junto a la mochila tipo macuto, abierta, en el andén de El Pozo frente al vagón número 3, otra bolsa a unos 10 metros. Esta segunda cerrada. El hecho se comunicó, sin que se sepa el destino de esa segunda bolsa cerrada (SAN 65/2007, f. 519). 
En estos casos puede observarse, en los fragmentos subrayados, el esfuerzo del juez redactor de la sentencia del $11 \mathrm{M}$ por dejar bien claras las referencias en su discurso, incluso cuando estas resultan fáciles de identificar, como ocurre en los dos ejemplos anteriores. En estos, los nombres 'tarjeta' y 'bolsa' que, además, repiten literalmente el antecedente, aparecen acompañados por modificadores que permiten identificar sin esfuerzo y de forma unívoca el antecedente.

Por una parte, el modificador 'última' del ejemplo (9) deshace la ambigüedad que podría darse con otro antecedente posible: el número de teléfono mencionado en el párrafo anterior. Aunque este está muy alejado para competir como posible antecedente y ni siquiera concuerda con la expresión anafórica 'esta última tarjeta', el juez prefiere no dar pie a interpretaciones ambiguas, e incluso añade una aclaración con la repetición literal del número de la tarjeta. Lo mismo ocurre con la bolsa del ejemplo (10), que se identifica con el numeral 'segunda' y también con un participio adjetival repetido ('cerrada').

En lo que se refiere a los otros sintagmas anafóricos empleados, con expresiones como 'dicho' o 'tal', cabe decir que las expresiones con 'dicho' son más empleadas por el juez del 11M que las construidas con 'tal': mientras que las primeras constituyen un $9 \%$ del total de expresiones anafóricas analizadas, las expresiones con 'tal' representan un 3,5\%. Se trata de una elección relevante, ya que, como se ha observado en el análisis cualitativo de los ejemplos, el determinante 'dicho/a/os/as', por su propio contenido metalingüístico, va a menudo seguido de repeticiones casi literales del antecedente, mientras que 'tal/es', cuyo significado "destaca la idea de cualidad o de modo" (Mederos, 1988: 81), suele preceder con mayor frecuencia a reformulaciones abstractas del antecedente. Los fragmentos (11) y (12) ejemplifican el uso de estos dos 'pseudo demostrativos' en la sentencia del 11M:

(11) Al día siguiente de los atentados, el 12 de marzo de 2004, el instructor dicta auto acordando el secreto de la actuaciones [sic.] por plazo de treinta días.

Dicho auto contiene en sus razonamientos una amplia justificación de la legalidad general de la medida, con cita de los preceptos legales aplicables y de la doctrina del Tribunal Constitucional y del Tribunal de Derechos Humanos aplicables al caso (SAN 65/2007, ff. 430-431).

(12) En definitiva, el tiempo de prolongación del secreto no es excesivo atendida la gravedad de los hechos y la complejidad de la investigación que hubiera perdido su eficacia de no haberse adoptado tal medida (SAN 65/2007, f. 441).

La observación anterior apunta a que el empleo del determinante 'dicho' con sustantivos que repiten literal o cuasiliteralmente el antecedente parece ser también menos costosa de iden- 
tificar y de interpretar unívocamente que el de expresiones equivalentes con 'tal', mucho más frecuentes en las otras sentencias de la A.N. analizadas (con un $21 \%$ de las ocurrencias).

Por último, cabe destacar que en ambos corpus el falso anafórico ‘el mismo’ / 'la misma' / 'los mismos' / 'las mismas' es el mecanismo de referencia retrospectiva menos empleado. No obstante, una vez más, su frecuencia de uso es mucho menor en la sentencia del $11 \mathrm{M}$ que en las sentencias de la A.N. (con el 1,5\% de las ocurrencias frente al 9\%). Pero, además, en los escasos casos en los que el juez del $11 \mathrm{M}$ emplea esta expresión, parece ser consciente de la ambigüedad que suelen comportar estas expresiones tan características del discurso jurídico e introduce aclaraciones que recuperan el antecedente, como ocurre en (13):

(13) Y si las partes lo que sostienen es que se han producido relevantes irregularidades en la cadena de custodia que priva a la bolsa con el explosivo de la condición de fuente de prueba (...) están aceptando la preexistencia del objeto (bolsa con explosivo) y su relación con el delito, aunque privado de valor probatorio.

El matiz, aparentemente irrelevante, no lo es.

- En el primer caso, las partes insinúan que la prueba no existe, haciendo innecesario el análisis de la cadena de custodia. A esto el Tribunal no tiene nada que decir:(...)

- En el segundo caso, por el contrario, admiten que la pieza de convicción existe, pero que las deficiencias en la custodia policial y en el control judicial de la misma -bolsa con el explosivo-, denotan irregularidades de tal entidad que impiden tener por cumplidas las garantías de identidad e integridad de la pieza (SAN 65/2007, f. 513).

\section{CONCLUSIONES}

Este análisis pretendía, por una parte, observar qué mecanismos discursivos empleaba el juez del $11 \mathrm{M}$ para que su sentencia no resultara ambigua en ningún punto y para que la interpretación del texto resultara accesible para receptores no expertos; y, por otra parte, pretendía también presentar una propuesta metodológica destinada a identificar las estrategias que potencian la claridad de un texto profesional.

Según la reciente teoría de la Lingüística de la Optimización, para mejorar la eficacia de los textos técnicos el analista debe cuestionarse si los rasgos propios de los géneros profesionales, que se perpetúan en el aprendizaje de la escritura experta por considerarse característicos de dichos géneros, son realmente eficaces para cumplir con los objetivos comunicativos de dichos textos (Göpferich, 2000). En esta línea, en este artículo planteamos, a partir del caso de un texto tradicionalmente complejo como la sentencia judicial, la posibilidad de desarrollar 
la optimización de discursos profesionales a partir del análisis de textos técnicos redactados de forma eficaz o con una cierta conciencia de la escritura del texto, como es el caso de la sentencia del $11 \mathrm{M}$.

Para poder efectuar un análisis más completo, nos hemos centrado en el estudio de las expresiones anafóricas, elemento esencial de la coherencia y cohesión del texto. El análisis de los resultados obtenidos sobre las expresiones demostrativas corrobora que, tal como indicaba la escasa bibliografía existente, en todas las sentencias estudiadas suele preferirse el sintagma anafórico, más informativo, al uso del pronombre demostrativo, que requiere un antecedente próximo y disponible para que la interpretación sea unívoca.

Asimismo, del contraste de la sentencia del $11 \mathrm{M}$ con otras sentencias dictadas por la Audiencia Nacional se desprende que, efectivamente, el juez del 11M parece prestar especial atención a la necesidad de asegurarse una interpretación correcta y clara de las expresiones anafóricas. El empleo eficaz de las expresiones demostrativas anafóricas en esta sentencia se observa principalmente en (i) una frecuencia mayor de empleo de los sintagmas anafóricos, que supera a la de las expresiones pronominales, aún más que en el resto de las sentencias examinadas; (ii) el empleo mayoritario del demostrativo y el determinante 'dicho/a/os/as', por encima de 'tal/ es', que suele combinarse con nombres más abstractos; (iii) el uso muy escaso del falso anafórico 'el mismo', que casi siempre acarrea ambigüedades en la identificación del antecedente.

Los datos cuantitativos son, por el momento, orientativos, ya que deberían contrastarse con un corpus más amplio. En cambio, es especialmente significativo el análisis cualitativo de fragmentos concretos de la sentencia del 11M, que muestra, como se ha apuntado aquí, un uso considerablemente claro o eficaz de las expresiones anafóricas. A lo largo del análisis hemos concretado en qué consiste tal uso, con recursos como la aclaración, la repetición del antecedente o el uso de modificadores numerales o clasificadores.

Resulta evidente que, para juzgar con mayor precisión la eficacia del empleo de las expresiones anafóricas en textos judiciales, sería necesario completar este análisis con un estudio de los ejemplos más detallado, que tuviera en cuenta aspectos como la distancia media del antecedente, en qué casos el nombre que acompaña al demostrativo es una etiqueta discursiva o un elemento encapsulador de un fragmento previo y en qué casos este nombre constituye una mera repetición literal del antecedente. Un estudio de este tipo permitiría explicar, por una parte, cuándo es necesario o preferible emplear un sintagma anafórico en lugar de un pronombre o qué condiciones de uso impone cada tipo de expresión anafórica para ser empleada de modo eficaz. No obstante, tales propósitos, que resultarían de interés también para otros discursos profesionales en los que la identificación unívoca de la referencia es crucial (como 
ocurre, por ejemplo, con los documentos instruccionales), exceden el objetivo de este artículo, por lo que los dejamos meramente enunciados para futuras investigaciones.

\section{REFERENCIAS BIBLIOGRÁFICAS}

Alcaraz, E. \& Hugues, B. (2002). El español jurídico. Barcelona: Ariel.

Álvarez Martínez, M. A. (1989). El pronombre Vol. I: Personales, artículo, demostrativos, posesivos. Madrid: Arco/Libros.

Álvarez de Mon y Rego, I. (2006). A contrastive study of encapsulation and prospection in written scientific text. En J. Flowerdew \& M. Gotti (Eds.), Studies in specialized discourse (pp. 21-40). Bern: Peter Lang.

Ariel, M. (1988). Referring and accessibility. Journal of Linguistics, 24, 65-87.

Ariel, M. (1990). Accessing noun-phrase antecedents. London/New York: Routledge.

Bhatia, V. (1993). Analysing genre. Language use in professional settings. London/New York: Longman.

Biber, D., Conrad, S. \& Reppen, R. (1998). Corpus linguistics. Investigating language structure and use. Cambridge: Cambridge University Press.

Campos, M. A. (2007). El lenguaje de las ciencias jurídicas: Nuevos retos y nuevas visiones. En E. Alcaraz, J. Mateo \& F. Yus (Eds.), Las lenguas profesionales y académicas (pp. 155165). Barcelona: Ariel / IULMA.

Conte, M. (1996). Anaphoric encapsulation. En N. de Mulder \& L. Tasmowski (Eds.), Coherence \& Anaphora (pp. 1-33). Belgian Journal of Linguistics, 10. Amsterdam: John Benjamins.

de Miguel, E. (2000). El texto jurídico-administrativo: Análisis de una orden ministerial. Círculo de Lingüística Aplicada a la Comunicación (CLAC), 4.

Eguren, L. J. (1999). Pronombres y adverbios demostrativos. Las relaciones deícticas. En I. Bosque \& V. Demonte (Dirs.), Gramática descriptiva de la lengua española (pp. 929-972). Madrid: Espasa Calpe.

Fernández Ramírez, S. (1987). Gramática española. 3.2. El pronombre. Madrid: Arco Libros.

Fernández Soriano, O. (1999). El pronombre personal. Formas y distribuciones. Pronombres átonos y tónicos. En I. Bosque \& V. Demonte (Dirs.), Gramática descriptiva de la lengua española (pp. 1209-1274). Madrid: Espasa Calpe.

Figueras, C. (1996). Semántica y pragmática de las expresiones anafóricas. En J. L. Cifuentes (Coord.), Estudios de lingüística cognitiva (pp. 61-76). Alicante: Universidad de Alicante.

Figueras, C. (2000). Las expresiones referenciales. En E. Montolío (Coord.), Manual práctico de escritura académica (pp. 17-76). Barcelona: Ariel. 
Flowerdew, J. (2003). Signalling nouns in discourse. English for Specific Purposes, 22, 329346.

Francis, G. (1986). Anaphoric nouns. Birmingham: English Language Research.

Francis, G. (1994). Labelling discourse: An aspect of nominal-group lexical cohesion. En M. Coulthard (Ed.), Advances in written text analysis (pp. 83-101). London: Routledge.

Gibbons, J. (2003). Forensic linguistics. An introduction to language in the Justice System. Oxford: Blackwell Publishing.

Göpferich, S. (2000). Analysing LSP genres (Text Types): From perpetuation to optimization in text(-type) linguistics. En A. Trosborg (Ed.), Analysing Professional Genres (pp. 395414). Amsterdam/Philadelphia: John Benjamins.

Goutsos, D. (1997). Modeling Discourse Topic: Sequential Relations and Strategies in Expository Text. Advances in Discourse Processes. New Jersey: Ablex Publishing Corporation.

Gülich, E. \& Kotschi, T. (1995). Discourse production in oral communication. En U. Quasthoff (Ed.), Aspects of oral communication (pp. 30-66). Berlin: Walter de Gruyter.

Gundel, J. K., Hedberg, N. \& Zacharski, R. (1993). Cognitive status and the form of referring expressions in discourse. Language, 69(2), 274-307.

Halliday, M. \& Hasan, R. (1976). Cohesion in English. London: Longman, 1985.

Ivanic, R. (1991). Nouns in search of a context: A study of nouns with both open- and closedsystem characteristics. Intenational Review of Applied Linguistics, 29(2), 93-114.

Kurzon, D. (1997). Legal language: Varieties, genres, registers, discourses. International Journal of Applied Linguistics, 7(2), 119-139.

López Samaniego, A. (2005). El uso metaargumentativo de las perífrasis obligativas en el lenguaje judicial español. En M. V. Calvi \& L. Chierichetti (Eds.), Nuevas tendencias en el discurso de especialidad (pp. 117-140). Bern: Peter Lang.

López Samaniego, A. (2006). Los ordenadores del discurso enumerativos en la sentencia judicial, ¿estrategia u obstáculo?. Revista de Llengua i Dret, 45, 61-87.

Lyons, J. (1980). Semántica. Barcelona: Teide.

Maia, B. (2003). What are comparable corpora? [en línea]. Disponible en: http://web. letras.p.pt/bhsmaia/belinda/pubs/CL2003\%20workshop.doc

Martínez Linares, M. A. (2006). Sobre la coordinación y la ambigüedad estructural en las sentencias. Estudios de lingüística, 20, 259-286.

Mederos, H. (1988). Procedimientos de cohesión en el español actual. Santa Cruz de Tenerife: Publicaciones del Cabildo Insular de Tenerife.

Montolío, E. (2001). Conectores de la lengua escrita. Barcelona: Ariel.

Montolío, E. \& López Samaniego, A. (2006). La propuesta didáctica en formación escrita llevada 
a cabo en la Escuela Judicial de España. En Actas del $V$ Congreso Internacional de AELFE (64-69). [CD-ROM] Versión Windows 95/98/2000/ Millenium/NP/XP. Zaragoza: Prensas Universitarias de Zaragoza.

Montolío, E. \& López Samaniego, A. (2008). La escritura en el quehacer judicial. Estado de la cuestión y presentación de la propuesta aplicada en la Escuela Judicial de España. Revista Signos, 41(66), 33-64.

Ordóñez, D. (2002). Lenguaje judicial: Argumentación y estilo. La Ley: Revista jurídica española de doctrina, jurisprudencia y bibliografía, 4, 1840-1854.

Portolés, J. (2004). Pragmática para hispanistas. Madrid: Síntesis.

Real Academia Española, Asociación de Academias de la Lengua Española (2005). Diccionario panhispánico de dudas. Madrid: Santillana.

Samaniego, E. (2005). El lenguaje jurídico: Peculiaridades del español jurídico. En P. Fuertes (Coord.), Lengua y sociedad: Investigaciones recientes en lingüistica aplicada (pp. 273310). Valladolid: Universidad de Valladolid.

Solan, L. M. (1993). The language of judges. Chicago: The University of Chicago Press.

Tadros, A. (1985). Prediction in text. Birmingham: English Language Research (University of Birmingham).

Tiersma, P. (1999). Legal language. Chicago/London: The University of Chicago Press.

van Dijk, T. (1978). La ciencia del texto. Barcelona/Buenos Aires: Paidós Comunicación.

von Heusinger, K. (2007). Accessibility and definite noun phrases. En M. Schwarz-Friesel, M. Consten \& M. Knees (Eds.), Anaphors in text. Cognitive, formal and applied approaches to anaphoric reference (pp. 123-144). Amsterdam/Philadelphia: John Benjamins.

\section{NOTAS}

1 Este trabajo se enmarca en el proyecto Análisis Lingüístico y Pragmático de la Recomendación Experta en Documentos de Ámbitos Profesionales (Referencia: FFI2008-00823), financiado por el Ministerio de Ciencia e Innovación, así como por los fondos FEDER. Deseo expresar mi más profundo agradecimiento a la Dra. Estrella Montolío, por haber impulsado la elaboración de este artículo, así como por sus certeras indicaciones y propuestas sobre su contenido.

2 Una versión preliminar de este trabajo se presentó en la IV SIS Conference Spanish at Work, que se celebró en Swansea (Reino Unido) en marzo de 2008. Agradezco a mi colega Raquel Taranilla sus valiosas ideas y sugerencias durante el proceso de elaboración de este artículo.

3 Se toman aquí las denominaciones para los oyentes propuestas por Portolés (2004) a partir de Goffman. Agradezco a un revisor anónimo la propuesta de aplicar estos conceptos a la caracterización de la 'doble audiencia' de la sentencia judicial.

4 Dado que la visión panorámica de tales movimientos no es el objetivo principal de esta comunicación, remitimos al lector a un artículo anterior (Montolío \& López Samaniego, 2008). 
5 Disponible en la página web del Ministerio de Justicia [Consulta 31/1/2007]: http: / /www.mjusticia.es/cs/Satellite?blobcol=urlpdf\&blobheader=application\%2Fpdf\&blobkey=id\&blobtable=AtC iudadanoPrincipal\&blobwhere=1068628785163\&ssbinary=true .

6 La teoría de la conspiración, mantenida por el Partido Popular y algunos medios de comunicación afines desde los días posteriores al atentado, defendía que la organización terrorista ETA había sido la inductora del atentado, que había respondido a una conspiración para evitar la victoria del Partido Popular en las elecciones del 14 de marzo, tres días después del atentado.

7 Nos referimos aquí, en rigor, a las sentencias de distintos órganos jurisdiccionales con las que hemos trabajado en López Samaniego, 2005, 2006; Montolío y López Samaniego, 2006, 2008.

8 Dada su extensión, en las referencias a la sentencia del $11 \mathrm{M}$ se incluye la referencia y el folio en el que aparece el fragmento citado. Las negritas, de no afirmarse lo contrario, son nuestras.

9 La ambigüedad causada por la coordinación ha sido recientemente analizada por Martínez Linares (2006) en el contexto de la sentencia judicial.

10 Una muestra de esta conciencia de los problemas de interpretación que puede acarrear el uso de pronombres es el hecho de que los juristas cuentan con principios que rigen la interpretación de los referentes, como el principio 'noscitur sociis', según el cual las palabras deben interpretarse de acuerdo con el contexto textual en el que aparecen (Gibbons, 2003); un principio que tiene su equivalente en la teoría lingüística general, según la cual los pronombres y las expresiones anafóricas en general solo pueden utilizarse en un escrito cuando en el contexto precedente puede identificarse claramente un solo referente posible (Figueras, 2000).

11 Estos nombres anafóricos han recibido distintos nombres, que implican diferencias de caracterización: general nouns (Halliday \& Hasan, 1976); anaphoric nouns o retrospective labels (Francis, 1986, 1994); carrier nouns (Ivanic, 1991); anaphoric encapsulations (Conte, 1996); encapsulating nominals (Goutsos, 1997); o signalling nouns (Flowerdew, 2003).

\section{CORPUS}

Westlaw.es. El servicio Internet de Aranzadi. Extraído el 15 de febrero de 2008 desde http:// www.westlaw.es/acceso/index.html

Ministerio de Justicia. Gabinete de prensa. SENTENCIA de la Sección segunda de la Sala de lo penal de la Audiencia Nacional, por los atentados ocurridos en Madrid el 11 de marzo de 2004. Extraído el 10 de diciembre de 2008 desde http://www.mjusticia.es/cs/Satellite?c=Noticia_S alaPrensa\&cid=1161678080871\&lang=es_es\&pagename=Portal_del_ciudadano\%2FNoticia_ SalaPrensa\%2FTplNoticia_SalaPrensa 